\title{
Conditional Sampling Analysis of Acoustic Phenomena from a Supersonic Jet Impinging on an Inclined Flat Plate*
}

\author{
Masahito AKamine, ${ }^{1 \dagger \dagger}$ Koji OKAmoto, ${ }^{1)}$ Susumu Teramoto, ${ }^{2)}$ Takeo OKUnUKI, ${ }^{2)}$ and Seiji TsuTsumi ${ }^{3)}$ \\ ${ }^{1)}$ Department of Advanced Energy, Graduate School of Frontier Sciences, The University of Tokyo, Kashiwa, Chiba 277-8561, Japan \\ ${ }^{2)}$ Department of Aeronautics and Astronautics, Graduate School of Engineering, The University of Tokyo, Tokyo 113-8656, Japan \\ ${ }^{3)}$ Aerospace Research and Development Directorate, Research Unit III, Japan Aerospace Exploration Agency, \\ Sagamihara, Kanagawa 252-5210, Japan
}

\begin{abstract}
Acoustic waves generated from an impingement of the exhaust jet on the flame deflector is one of the causes of the payload vibration during the liftoff of a launch vehicle. To understand this acoustic phenomenon, the authors conducted experiments using a correctly expanded jet impinging on an inclined flat plate, and observed an acoustic wave propagating from the jet impingement region in a direction approximately perpendicular to the plate. The results of the wavelet analysis indicated that this acoustic phenomenon occurs intermittently. In this study, a flow phenomenon related to this intermittent acoustic phenomenon is extracted from schlieren visualization movies and wall surface pressure signals using a conditional sampling analysis. The extracted flow phenomenon is similar to the Mach wave of a free jet. With the nozzle-plate distance at which the OASPL of this acoustic phenomenon is the maximum, the flow phenomenon extracted is also similar, but some minor differences appear. These differences are expected to be a key to understand the change in OASPL when varying the nozzle-plate distance.
\end{abstract}

Key Words: Acoustics, Supersonic Jet Noise, Schlieren Visualization, Wavelet Analysis, Conditional Sampling Analysis

\section{Introduction}

One of the causes of payload vibration during liftoff of a launch vehicle is the acoustic waves from the exhaust jet, which impinges on a flame deflector. These acoustic waves were considered to be generated only from the sources along the jet in NASA SP-8072 ${ }^{1)}$ and its modifications. ${ }^{2,3)}$ However, a numerical simulation by Tsutsumi et al. ${ }^{4)}$ showed that the acoustic field at a launch pad changes significantly with a slight modification of deflector geometry, and inferred that acoustic waves from the jet impingement region will affect the acoustic field. The deflector is required not to generate these acoustic waves in the direction of the launch vehicle. To establish the design criteria, it is essential to understand the mechanism of how acoustic waves are generated from the supersonic impinging jet.

The flow phenomena of the supersonic impinging jet have been studied for the last five decades after Henderson ${ }^{5)}$ using the jet impinging on a perpendicular or inclined flat plate. In Donaldson and Snedeker ${ }^{6)}$ and subsequent studies, it was described that the flow field consisted of the free jet, impingement, and wall jet regions. Donaldson and Snedeker ${ }^{6)}$ observed a recirculation flow in the impingement region, and Carling and Hunt ${ }^{7)}$ discussed a structure of expansion, compression, and shock waves on the plate surface formed by rapid expansion of the jet after impingement. Additionally, the experiments by Lamont and Hunt ${ }^{8)}$ and Nakai et al. ${ }^{9)}$

(C) 2016 The Japan Society for Aeronautical and Space Sciences

*Received 31 July 2015; final revision received 1 April 2016; accepted for publication 25 April 2016.

†Corresponding author, akamine@ thermo.t.u-tokyo.ac.jp and the numerical simulation by Goto et al., ${ }^{10)}$ showed the complicated shock structure in an underexpanded jet impinging on an inclined flat plate.

Many studies on the acoustic phenomena of a supersonic jet impinging on a perpendicular plate have also been conducted, and most of them concerned the discrete tone. Powell ${ }^{11)}$ suggested the model that an acoustic wave from the impingement region made a disturbance at the nozzle exit, and formed a feedback loop. There are many extensive studies on this feedback loop, especially phenomena in the free jet region (e.g., Krothapalli et al. ${ }^{12)}$ and Uzun et al. ${ }^{13)}$ ) and in the impingement region (e.g., Henderson et al., ${ }^{14)}$ Dauptain et al., ${ }^{15)}$ and Risborg and Soria ${ }^{16)}$ ).

Broadband acoustic phenomena are important during the liftoff of a launch vehicle. Nonomura et al. ${ }^{17)}$ and Tsutsumi et al. ${ }^{18,19)}$ conducted numerical simulations using a correctly expanded jet impinging on an inclined flat plate, and predicted an acoustic wave from the impingement region, which propagated in a direction approximately perpendicular to the plate, as well as the Mach wave from the free and wall jet regions, as shown in Fig. 1. Moreover, because the source of the acoustic wave from the impingement region was close to a shock structure in the impingement region, it was suggested that this acoustic wave was the shock-associated noise. As for experimental studies, only Worden et al., ${ }^{20)}$ in which sound-pressure-level (SPL) spectra were measured at two fixed locations using a similar impinging jet, can be found.

The authors ${ }^{21)}$ have experimentally studied the characteristics of the acoustic wave, such as the propagation direction and location of the source region. In this experiment, a Mach 


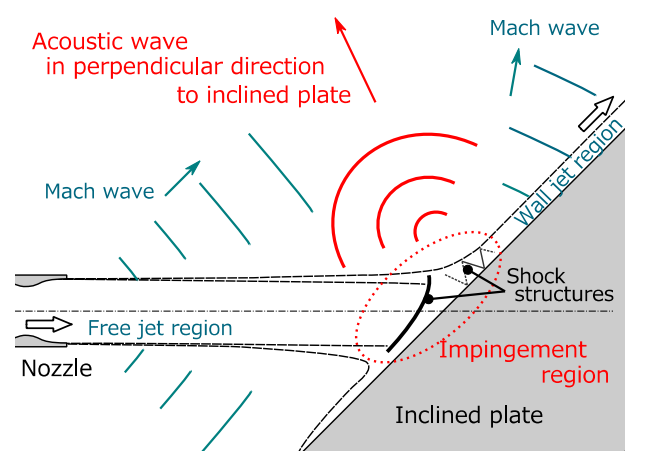

Fig. 1. Schematic overview of acoustic waves generated from an impinging jet.

1.8 correctly expanded jet impinged on a $45^{\circ}$ inclined flat plate (nozzle exit diameter $D=20 \mathrm{~mm}$; nozzle-plate distance $5 D$ ), and measurements of the spatial SPL distribution and schlieren visualization were carried out. The results showed two types of acoustic waves, as suggested by Tsutsumi et al. ${ }^{18)}$ In particular, as for the acoustic wave from the impingement region, it was indicated that the source region was concentrated and located near the shock structures, from an SPL decay by the distance from the source and frequency analysis of the visualization movie.

Moreover, the authors ${ }^{22)}$ examined whether or not the generation of this acoustic wave is related to the shock structure in the impingement region, as suggested by Tsutsumi et al., ${ }^{18)}$ varying the nozzle-plate distance from $5 D$ to $15 D$. The OASPL in the $15 D$ case was higher than that in the $5 D$ case. However, the shock structure becomes weaker with the longer nozzle-plate distance. Therefore, this acoustic wave cannot be explained as shock-associated noise only.

The authors ${ }^{23)}$ also discovered that this acoustic phenomenon occurred intermittently from the results of a wavelet analysis. Because detailed features of this phenomenon were not clarified from the results of the conventional FFT analysis, the authors proposed a new approach using a conditional sampling method. The intermittent acoustic phenomena have mainly been studied using a subsonic free jet with several approaches such as: a method based on Lighthill's acoustic analogy by Juvé et al. ${ }^{24)}$; a conditional sampling method using amplitude peaks of a far-field acoustic signal as a trigger by Guj et al. ${ }^{25)}$; a combination of a wavelet analysis of farfield acoustic signals and a proper orthogonal decomposition (POD) of images of the large-scale turbulent structures visualized using light scattering due to the moisture condensation by Hileman et al. ${ }^{26)}$; and a statistical analysis of the acoustic events by Kearney-Fischer et al. ${ }^{27)}$ The authors' method $^{23)}$ is similar to that of Guj et al. ${ }^{25)}$ but differs in the use of wavelet analysis for trigger detection, which enables us to divide the phenomenon into each frequency component.

The objective of this study is to discuss the characteristics of the flow phenomenon related to generation of the acoustic wave from the impingement region, which is extracted using the conditional sampling method proposed by the authors. ${ }^{23)}$
Table 1. Experimental condition.

\begin{tabular}{lcc} 
Jet Mach number & $M_{j}$ & 1.8 \\
Nozzle exit diameter, mm & $D$ & 20 \\
Stagnation pressure, $\mathrm{MPa}$ & & $0.585 \pm 0.01$ \\
Stagnation temperature, K & & 300 \\
Reynolds number & $\operatorname{Re}_{D}$ & $1.5 \times 10^{6}$ \\
Nozzle-plate distance, $D$ & & 5,15 \\
Plate angle, deg & & 45 \\
Convective Mach number & & 0.79 \\
\hline
\end{tabular}

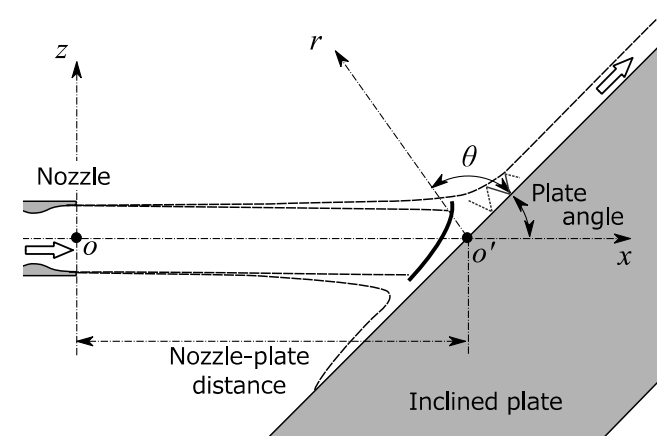

Fig. 2. Coordinate system.

After explaining the methods of the experiment and analysis in Sec. 2, the results extracted in the case of the nozzle-plate distance $5 D$ are described in Sec. 3.1. Then, the flow phenomenon extracted is discussed in Sec. 3.2. Finally, the change in features extracted using different nozzle-plate distances is shown in Sec. 3.3.

\section{Method of Experiment and Analysis}

\subsection{Experimental method}

The present experiment is carried out using a jet facility at the Hypersonic High-enthalpy Wind Tunnel at the Kashiwa Campus of The University of Tokyo. The experimental conditions and coordinate systems are shown in Table 1 and Fig. 2, respectively. A Mach 1.8 correctly expanded jet from a nozzle impinges on a $45^{\circ}$ inclined flat plate. The nozzleplate distance is set at $5 D$ for Sec. 3.1 and $15 D$ for Sec. 3.3. Detailed explanations of the jet facility, geometries of the nozzle and inclined plate, Mach number distribution on the centerline, and acoustic environment are shown in Akamine et al. ${ }^{21)}$

\subsection{Measurements}

In this study, for the conditional sampling analysis, acoustic measurement using a microphone and flow measurement are carried out synchronously. The flow measurement consists of schlieren visualization and wall surface pressure measurement on the inclined plate. To measure the acoustic wave from the impingement region, which propagates in a direction approximately perpendicular to the inclined plate, a microphone is set at $r / D=40, \theta=75^{\circ}$. The visualization movies of the flow field between the nozzle and impingement region are taken by a high-speed camera in two (in the case of the nozzle-plate distance $5 D$ ) and five divided parts (in $15 D$ case). Additionally, to measure the wall surface pressure 


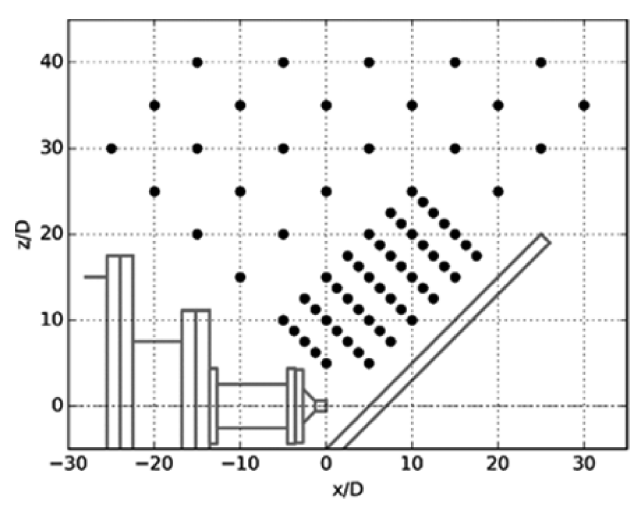

Fig. 3. Measurement points of OASPL (nozzle-plate distance, 5D).

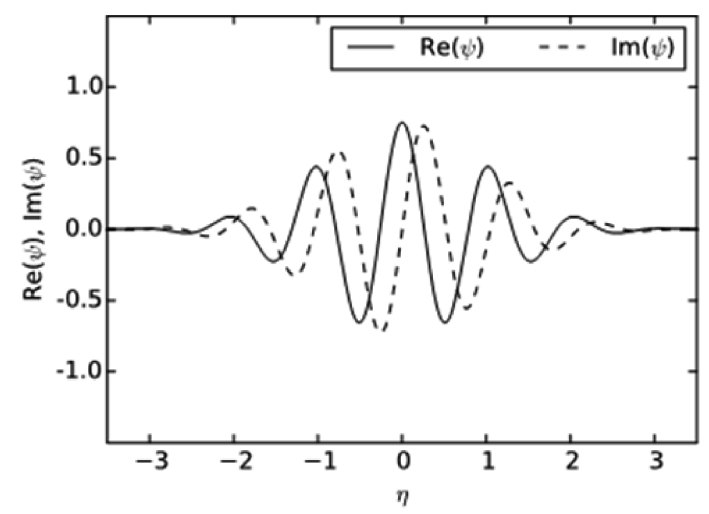

Fig. 4. Morlet function $\left(\omega_{0}=6\right)$. fluctuation in the impingement region, a pressure transducer is mounted at $r / D=0.1\left(\theta=0^{\circ}\right.$, i.e., flush-mounted on the plate surface).

A Brüel \& Kjær 1/4" free-field microphone type 4939 and a NEXUS microphone conditioner type 2690 are used for acoustic measurements, and a Kulite XCQ-062-7-BAR-A pressure transducer $(\phi=1.7 \mathrm{~mm})$ and a unipulse AM30 amplifier are used for the wall surface pressure measurement. These signals are logged using a National Instruments DAQ PCI-6133 and LabView (sampling frequency, $400 \mathrm{kHz}$; duration, $1 \mathrm{~s}$ ).

Schlieren visualization movies are taken using an optical system that comprised of a mercury lamp, pair of concave mirrors ( $\phi=200 \mathrm{~mm}$; focal length, $2 \mathrm{~m}$ ), knife edge (perpendicular to the jet axis), and a Photron FASTCAM SA-Z high-speed camera (exposure time, approximately $0.37 \mu \mathrm{s}$; frame rate, $100 \mathrm{kHz}$; duration, $1 \mathrm{~s}$; resolution, $640 \times 280$ or $384 \times 480$ pixels).

To determine the microphone location, the overall soundpressure-level (OASPL) was measured while moving a single microphone using a two-dimensional positioning system. The locations of measurement points relative to the inclined plate are fixed, when the nozzle-plate distance is changed. The measurement points in the $5 D$ case are shown in Fig. 3. During the measurement, the microphone points at $o^{\prime}$ in Fig. 2 (the intersection point between the jet axis and plate surface) within $30^{\circ}$, which causes less than $1.7 \mathrm{~dB}$ deviation $(0.088 \mathrm{~dB}$ at $5 \mathrm{kHz}, 0.41 \mathrm{~dB}$ at $15 \mathrm{kHz}$, and $1.7 \mathrm{~dB}$ at $40 \mathrm{kHz})$. To calculate the SPL, the microphone free-field response of incidence angle $0^{\circ}$ and atmospheric absorption are corrected as described in Akamine et al. ${ }^{21)}$

\subsection{Conditional sampling analysis}

\subsubsection{Overview}

A flow phenomenon related to the intermittent generation of the acoustic wave is extracted from the visualization movie or wall surface pressure signal using the following analysis. An intermittent amplitude peak of each frequency component (i.e., "trigger event") is detected by a wavelet transform of the far-field acoustic signal. Visualization images or wall surface pressure values in a certain interval around the trigger events are sampled and ensemble-averaged. By taking an ensemble average, it is expected that the flow phenomena related to this trigger event will be emphasized, whereas unrelated flow phenomena will be cancelled.

\subsubsection{Wavelet transform}

Complex values $\hat{p}\left(f, t_{n}\right)$ are calculated from a far-field acoustic signal $p\left(t_{n}\right)$ for each frequency $f$ using the following equation of the continuous wavelet transform ${ }^{28)}$ :

$$
\hat{p}\left(f, t_{n}\right)=\sum_{n^{\prime}=0}^{N-1} p\left(t_{n^{\prime}}\right) \psi^{*}\left[\frac{\left(t_{n}-t_{n^{\prime}}\right)}{s(f)}\right],
$$

where $t_{n}=n \Delta t$ is a time with $n=0,1, \ldots, N-1$ for a signal length $N=400 \times 10^{3}$ and $\Delta t=1 / 400[\mathrm{kHz}]=2.5 \mu \mathrm{s}$.

The Morlet function $\psi(\eta)=\pi^{-1 / 4} e^{-\eta^{2} / 2} e^{i \omega_{0} \eta}$ is a function that is a product of a sinusoidal wave and a Gaussian window function. Figure 4 shows the Morlet function in this analysis, with the parameter concerning the window width $\omega_{0}=6$, involving a scale factor

$$
s(f)=\left(\omega_{0}+\sqrt{2+\omega_{0}^{2}}\right) / 4 \pi f \approx 0.968 / f .
$$

In Eq. (1), the term $\psi\left[\left(t_{n}-t_{n^{\prime}}\right) / s(f)\right]$ is the Morlet function of a frequency $f$ at time $t_{n^{\prime}}$, and $\psi^{*}$ is the complex conjugate of $\psi$. Therefore, Eq. (1) shows the calculation of an inner product (i.e., a degree of similarity) between the farfield acoustic signal and the Morlet function of $f$ at $t_{n^{\prime}}$. The amplitude

$$
\left|\hat{p}\left(f, t_{n}\right)\right|=\sqrt{\operatorname{Re}(\hat{p})^{2}+\operatorname{Im}(\hat{p})^{2}}
$$

corresponds to the degree of similarity, and

$$
\arg \left(\hat{p}\left(f, t_{n}\right)\right)=\arctan (\operatorname{Re}(\hat{p}) / \operatorname{Im}(\hat{p}))
$$

represents the phase, which varies from $-\pi$ to $\pi$ in a period. 2.3.3. Trigger event detection and conditional sampling

A typical far-field acoustic signal $p\left(t_{n}\right) / \sigma_{p}$ and its amplitude $\left|\hat{p}\left(f, t_{n}\right)\right| / \sigma_{p}$ are shown in Figs. 5(a) and 5(b), respectively, where $\sigma_{p}$ is the root mean square of $p\left(t_{n}\right)$. In Fig. 5(b), for example, the $f=15 \mathrm{kHz}$ component is indicated by the red line, and its phase $\arg \left(\hat{p}\left(f, t_{n}\right)\right)$ is shown in Fig. 5(c). The amplitude peaks, which are indicated by red points in Fig. 5(b), are regarded as the arrival of the $15 \mathrm{kHz}$ acoustic 


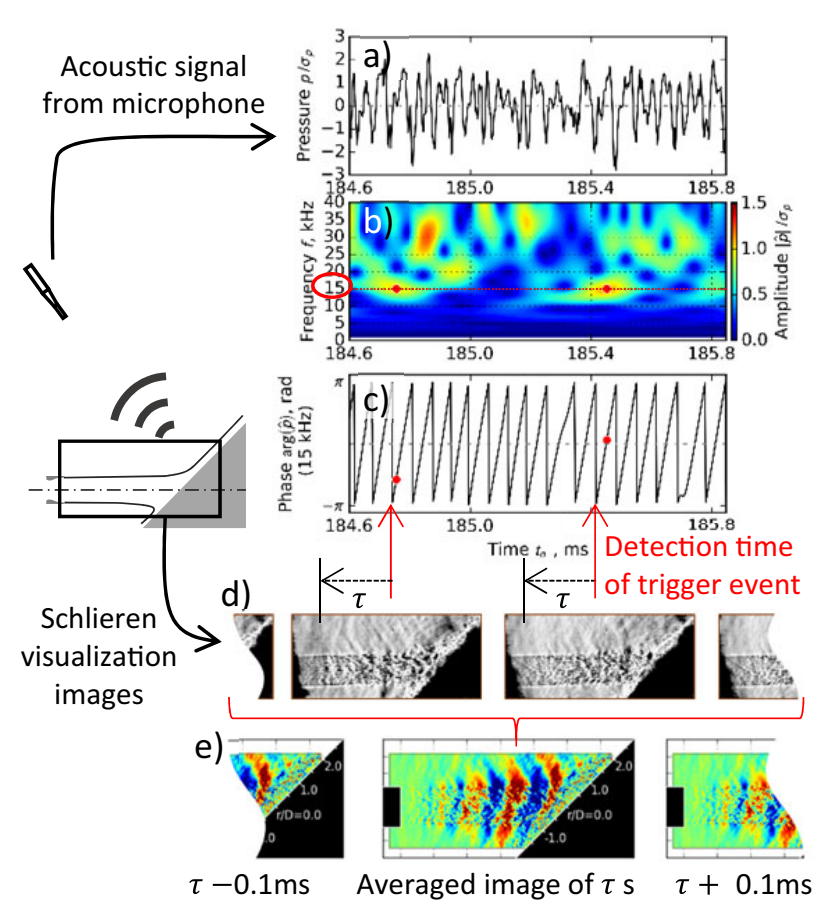

Fig. 5. Trigger event detection and conditional sampling.

wave to the microphone. The phase values of these amplitude peaks are not constant, as seen in Fig. 5(c). Therefore, the detection time of the trigger event is defined as the time at the same phase value $\arg \left(\hat{p}\left(f, t_{n}\right)\right)=-\pi$ to align the trigger events in phase. Note that the trigger events of $\left|\hat{p}\left(f, t_{n}\right)\right| \geq 0.4 \sigma_{p}$ are used here to eliminate the extremely weak peaks.

Depending on the detection time obtained, the visualization image or wall surface pressure value of a time $\tau$ before the detection time of the trigger event can be taken (Fig. 5(d)), and the average of them can be calculated. By varying $\tau$, a visualization movie or wall surface pressure waveform of the flow phenomenon related to the trigger event (i.e., the intermittent generation of the acoustic wave) can be extracted (Fig. 5(e)).

\section{Results and Discussion}

\subsection{Intermittent generation of acoustic wave and re- lated flow phenomenon}

As discussed in the previous study, ${ }^{21)}$ two types of acoustic wave are generated from the impingement region of the supersonic jet impinging on the inclined flat plate. Figure 6(a) shows an OASPL distribution, which was obtained by remeasurement conducted by Akamine et al. ${ }^{21)}$ This figure shows two high-OASPL regions due to two different acoustic waves. One propagates $30^{\circ}$ from the plate surface, and is explained as the Mach wave from the wall jet (flow along the plate surface after impingement) by numerical studies. ${ }^{17-19)}$ The other is acoustic wave propagating $75^{\circ}$ from the plate surface. Because its generation mechanism has not been clarified yet, this acoustic wave is the main focus of this study.

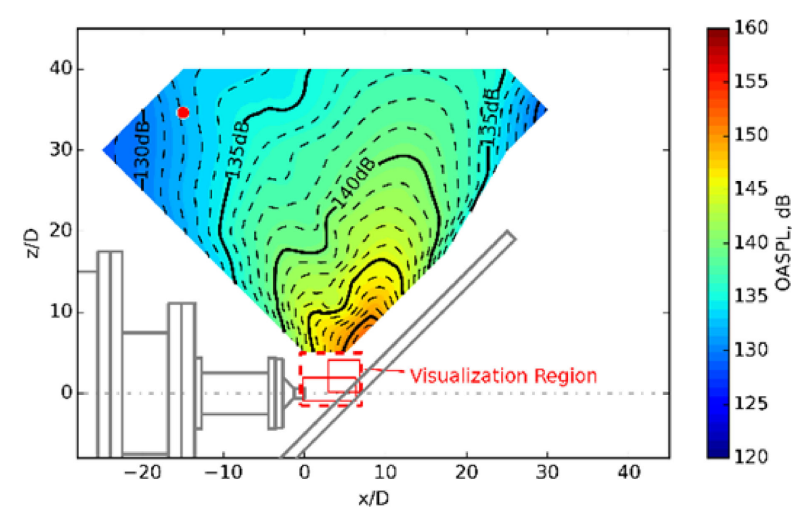

(a) Visualization region and OASPL distribution (red point, trigger microphone location, $r / D=40, \theta=75^{\circ}$ )

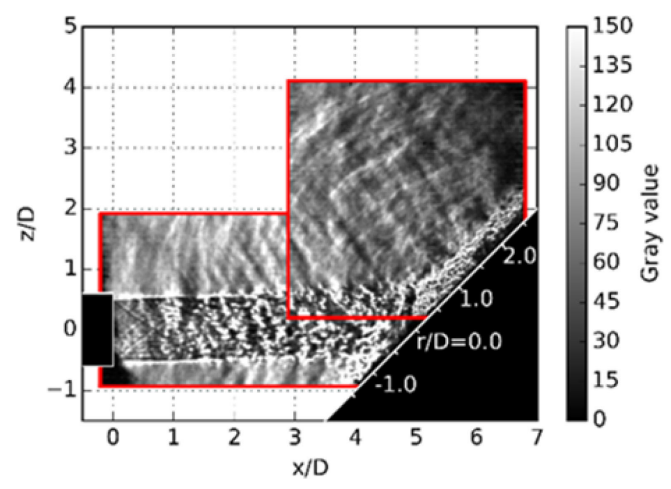

(b) Snapshot of the raw visualization movies

Fig. 6. Visualization movies in the case of a nozzle-plate distance of $5 D$.

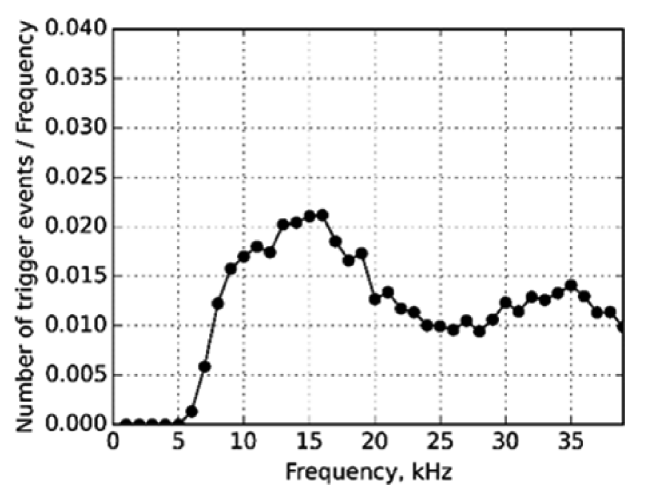

Fig. 7. Number of trigger event detections normalized by frequency $\left(r / D=40, \theta=75^{\circ}\right.$; nozzle-plate distance, $\left.5 D\right)$.

To extract a flow phenomenon that correlates to this acoustic wave, conditional sampling analysis is applied to the visualization movie. The trigger events are detected from the far-field acoustic signal measured using a microphone at $r / D=40, \theta=75^{\circ}$ (a red point in Fig. 6(a)). The visualization regions are the red squares in Fig. 6(a). A snapshot of the raw movies is shown in Fig. 6(b). The nozzle-plate distance is $5 D$.

Figure 7 shows the number of trigger events for each frequency component in the acoustic signal per second. The number of trigger events is normalized by frequency (i.e., number of periods included in a sine wave per second). This 


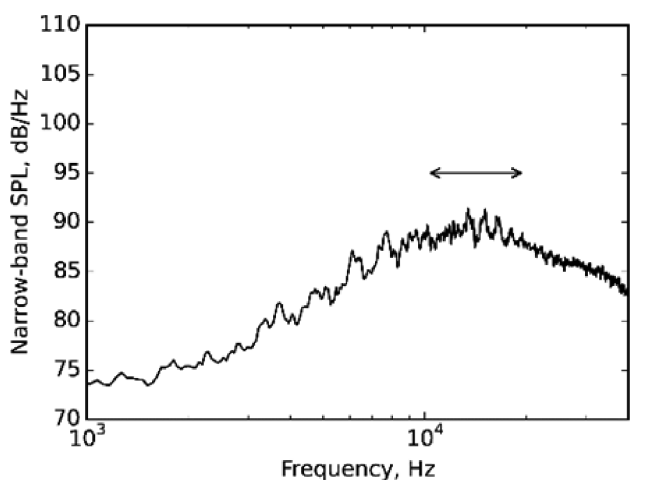

Fig. 8. FFT spectrum showing a broadband peak at $10-20 \mathrm{kHz}$ $\left(r / D=40, \theta=75^{\circ}\right.$; nozzle-plate distance, $\left.5 D\right)$.

normalization is needed to compare the number of trigger events between different frequencies because higher-frequency (shorter-period) components occur more frequently than lower-frequency (longer-period) components in the same-length signal. The peak frequency is about $15 \mathrm{kHz}$, and the peak frequency of the FFT spectrum of this acoustic signal is a similar frequency $(10-20 \mathrm{kHz})$, as shown in Fig. 8. Therefore, the $15 \mathrm{kHz}$ component is chosen for the discussion in this section.

Figures 9(a) and 9(b) show two of the images extracted from different visualization movies, using 289 trigger events of the $15 \mathrm{kHz}$ component. The green color in the contours indicates the time-averaged gray value, and red and blue indicate lighter and darker than that, respectively. It can be observed that extracted density fluctuation passes through the boundaries between the two different movies, indicating that the extracted results have reproducibility.

Figure 9(b) shows the image extracted at $\tau=-2.20 \mathrm{~ms}$ ( $2.20 \mathrm{~ms}$ before the detection time of the trigger event; i.e., the arrival of the intermittent $15 \mathrm{kHz}$ acoustic wave at a microphone). Waves propagating from a source at $r / D \approx 1.0$ on the plate surface in a direction approximately perpendicular to the plate (Wave [1]) are observed. Wave [1] is observed to be generated at $\tau \approx-2.35 \mathrm{~ms}$ from the source. The distance between the source and microphone is $40 \mathrm{D}$ (=0.8 m). Therefore, the propagation velocity is calculated as approximately $340 \mathrm{~m} / \mathrm{s}$, which is almost equal to the sound speed of the surroundings. This indicates that Wave [1] is the acoustic wave detected at the microphone. Other waves (waves [2] and [3]) are also observed, and more careful investigation is necessary to discuss them.

In the images before the generation of these waves, a density fluctuation in the free jet region is extracted. For example, in the image at $\tau=-2.40 \mathrm{~ms}$ (Fig. 9(a)), the density fluctuation that moves along the jet to the inclined plate appears mainly at the upper side of the jet. In particular, this fluctuation appears not only outside of the jet, but also in the jet. Because the schlieren method does not give information on the location of density fluctuation along the light path, an wall surface pressure waveform extracted at $r / D=0.1$ (in the impingement region) is shown in Fig. 10. At $\tau \approx-2.40 \mathrm{~ms}$, when this fluctuation reaches

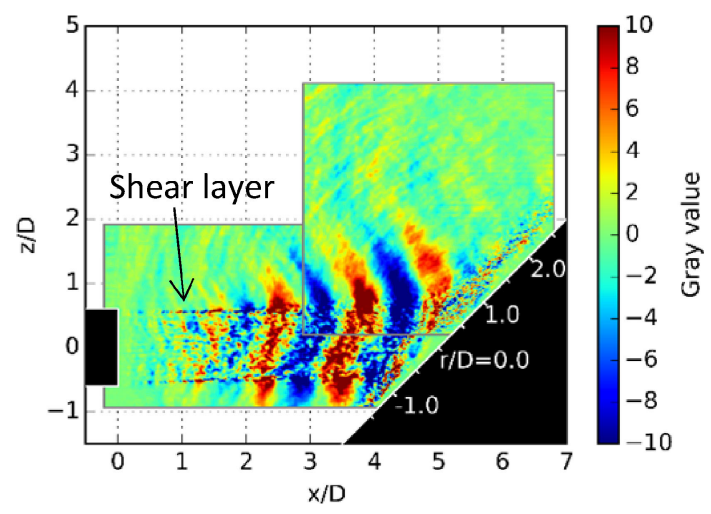

(a) $\tau=-2.40 \mathrm{~ms}$

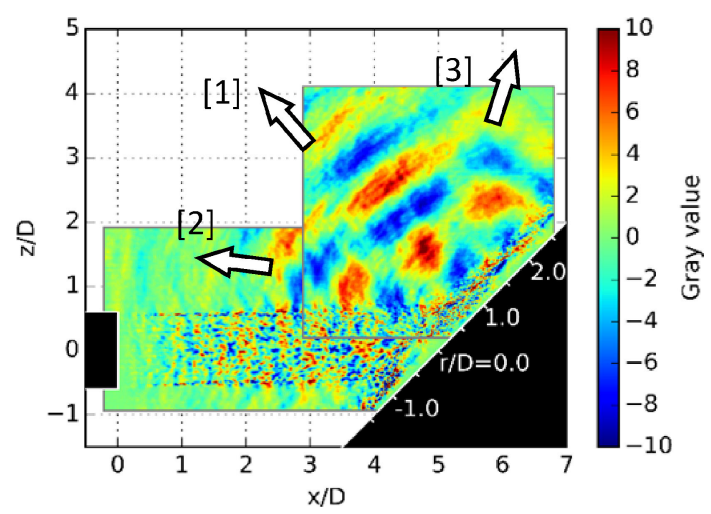

(b) $\tau=-2.20 \mathrm{~ms}$

Fig. 9. Extracted images of flow phenomena related to the acoustic waves from the visualization movie using 289 trigger events of $15 \mathrm{kHz}$ (nozzleplate distance, $5 D$ ).

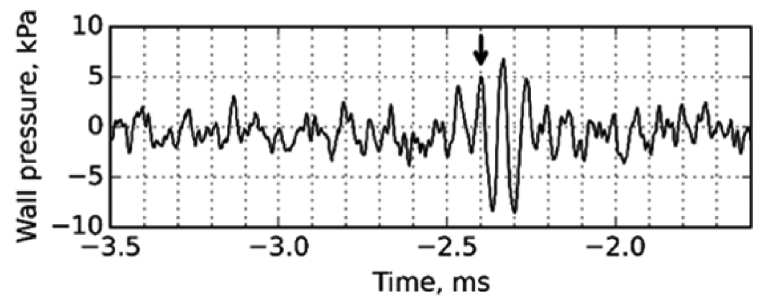

Fig. 10. Extracted wall surface pressure waveform at $r / D=0.1$ using 289 trigger events of $15 \mathrm{kHz}$ (nozzle-plate distance, $5 D$ ).

the inclined plate in Fig. 9(a), a pressure fluctuation with a large amplitude appears. Therefore, the flow phenomenon that consists of the density fluctuation at the upper side of the jet and pressure fluctuation in the jet relates to the intermittent generation of the acoustic wave from the impingement region.

\subsection{The extracted flow phenomenon and the Mach wave of the free jet}

The feature of the flow phenomenon extracted in Fig. 9(a) is similar to that of the Mach wave of the free jet. The Mach wave is explained by Oertel et al. ${ }^{29)}$ as a pressure wave generated by a supersonic convection of large-scale turbulent structures in the shear layer. It is hypothesized that the density fluctuation at outside of the jet is the Mach wave and the pressure fluctuation in the jet is generated by the large-scale 

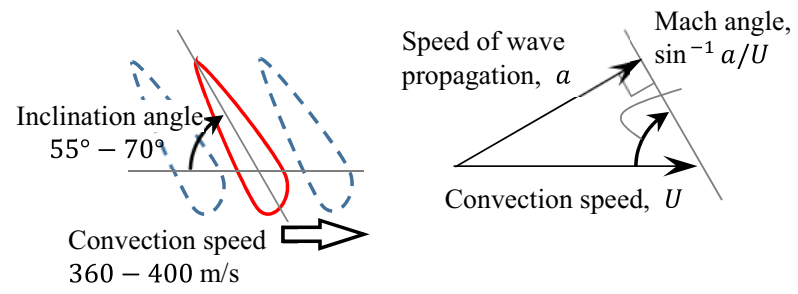

Fig. 11. Inclination angle and convection speed of density fluctuation extracted (left) and Mach angle (right).

turbulent structures. Based on this hypothesis, the angle of the density fluctuation to the jet axis will be the Mach angle for the convection speed. In Fig. 9(a) and other frames of the extraction movies, the inclination angle of these density fluctuations is approximately $55-70^{\circ}$. The convection speed of these density fluctuations is also estimated at $360-400 \mathrm{~m} / \mathrm{s}$ by tracking peaks of the gray values along the jet axis in the region of $z / D=0.7-1.2$ of the images extracted. These values are summarized in Fig. 11 left. As shown in Fig. 11 right, the Mach angle is calculated as $\sin ^{-1} a / U$, where $a$ is the speed of wave propagation and $U$ is the convection speed. If the speed of wave propagation is assumed to be the speed of sound in the surroundings (i.e., $a=340 \mathrm{~m} / \mathrm{s}$ ), the Mach angle for the convection speed $U=360-400 \mathrm{~m} / \mathrm{s}$ is approximately $60-70^{\circ}$, which almost corresponds to the inclination angle observed in the extraction movies. Therefore, this is considered to support the above hypothesis.

Moreover, if the hypothesis is true, a similar flow structure will also be extracted in the free jet of the same Mach number. To confirm this, an experiment for the free jet was carried out with the same nozzle and flow conditions, and the same measurement and analysis were applied. In this experiment, the microphone was set as shown in Fig. 12(a) to detect the trigger event from the far-field acoustic signal. In the results shown in Fig. 12(b), which were extracted using 270 trigger events of the $15 \mathrm{kHz}$ component, the shape of the density fluctuation extracted is similar to that in Fig. 9(a). Therefore, the hypothesis is also supported by these results. Additionally, in Fig. 12(b), the density fluctuation at the upper side of the jet is extracted more clearly than that at the lower side. This indicates that the azimuthal correlation length is short, and it is consistent with the results of the numerical simulation of the Mach wave by Freund et al. ${ }^{30)}$ Depending on this consideration, it is supposed that the density fluctuation extracted is the Mach wave of the free jet.

Finally, it is considered how the density fluctuation extracted shown in Fig. 9(a) generates the acoustic wave from the impingement region. The reflection of the Mach wave from the free jet region is likely to occur. However, the source region of the acoustic wave from the impingement region is concentrated, as reported by Akamine et al. ${ }^{21)}$ and also shown in Fig. 9(b). This feature does not appear only by the reflection. Therefore, there will be another mechanism of acoustic wave generation in the impingement region, and further investigation is necessary to clarify the whole mechanism.

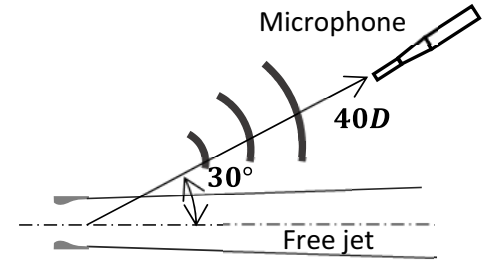

(a) Microphone location

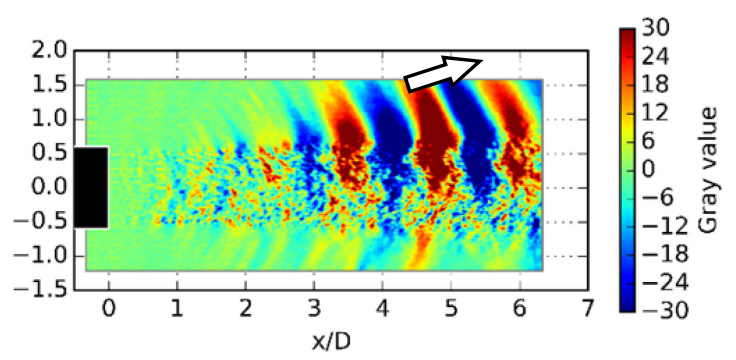

(b) Flow phenomena image extracted using 270 trigger events of $15 \mathrm{kHz}(\tau=-2.11 \mathrm{~ms})$

Fig. 12. Density fluctuation in the free jet case.

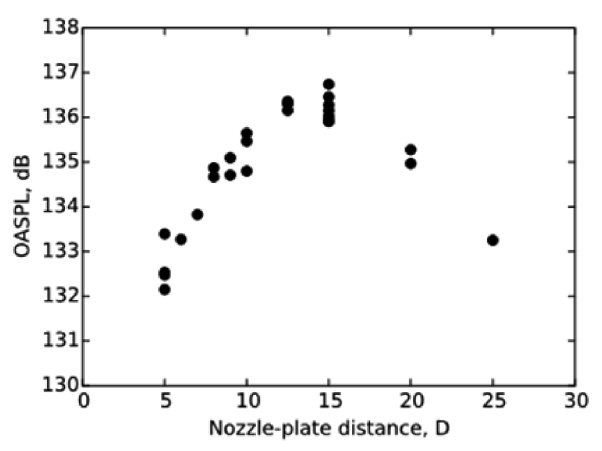

Fig. 13. OASPL as a function of the nozzle-plate distance $(r / D=40$, $\left.\theta=75^{\circ}\right)$

\subsection{Effect of the nozzle-plate distance}

As reported in Akamine et al., ${ }^{22)}$ the OASPL of the acoustic wave from the impingement region was higher with the nozzle-plate distance $15 D$ than that of $5 D$. Figure 13 shows that the maximum OASPL is measured when the nozzleplate distance is approximately $15 \mathrm{D}$. To investigate the cause of this characteristic, a density fluctuation extracted in the $15 D$ case is compared with that in the $5 D$ case.

In the $15 D$ case, both the peak frequency of the FFT spectrum and that of the number of trigger events are $7 \mathrm{kHz}$. Using 300 trigger events of the $7 \mathrm{kHz}$ component, density fluctuation is extracted from the visualization movies. The whole visualized region is covered by five frames of movies, as shown in Fig. 14. The results shown in Fig. 15 are similar to Fig. 9 (the $5 D$ case). After arrival of the density fluctuation at the inclined plate (Fig. 15(a); $\tau=-2.40 \mathrm{~ms}$ ), an acoustic wave is generated and propagates in the microphone direction (Fig. 15(b); $\tau=-2.00 \mathrm{~ms}$ ). Therefore, it is considered that the flow phenomenon related to acoustic wave generation is basically similar regardless of the nozzle-plate distance. 


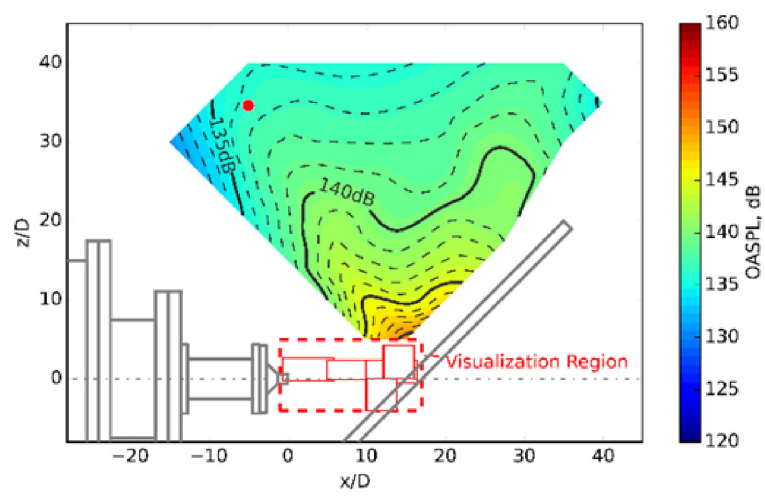

(a) Visualization region and OASPL distribution (red point, trigger microphone location, $r / D=40, \theta=75^{\circ}$ )

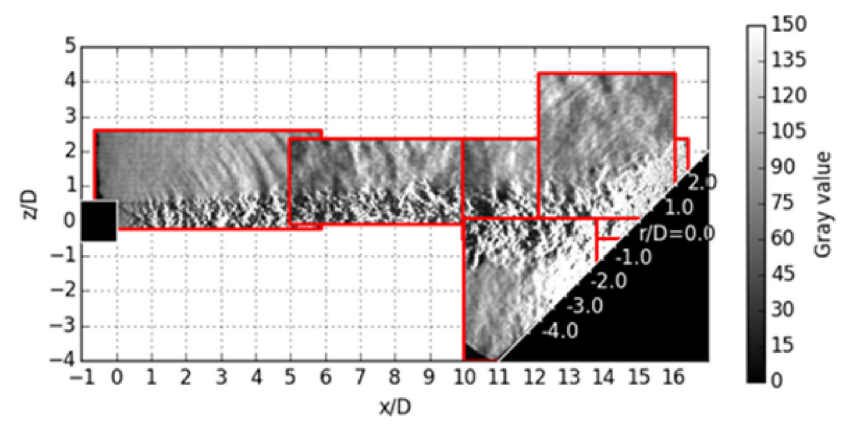

(b) Snapshot of the raw visualization movies

Fig. 14. Visualization movies in the case of the nozzle-plate distance of $15 D$.

However, some minor differences can be found. In Fig. 15(a), the density fluctuation at the lower side of the jet is mainly extracted, whereas that at the upper side is extracted in Fig. 9(a) (the 5D case). Additionally, a straight wave (indicated as Wave [4]) can be observed in Fig. 15(b). This is radiated from the region beneath the impingement region and propagates in a direction almost perpendicular to the plate surface. In the $5 D$ case (Fig. 9(b)), Wave [2] was observed in a similar region. However, Wave [2] has an arc-shaped wave front, is radiated from the upper side of the impingement region, and propagates in the upstream direction. Therefore, waves [2] and [4] are considered to be different acoustic phenomena. As for Wave [3], the corresponding region is not included in the present visualization region in the $15 \mathrm{D}$ case because the main focus of the present study is the acoustic wave propagating $75^{\circ}$ from the plate surface. Therefore, its existence is not discussed in this study. Understanding these differences will help clarify the relation between the OASPL and nozzle-plate distance in Fig. 13, and further studies are necessary.

\section{Conclusion}

In this study, experiments were carried out to discuss the features of flow phenomenon related to acoustic wave generation in a supersonic impinging jet. Because the acoustic wave was generated intermittently, a conditional sampling analysis was adopted. The flow phenomenon, which is related to the acoustic wave from the impinging region propa-

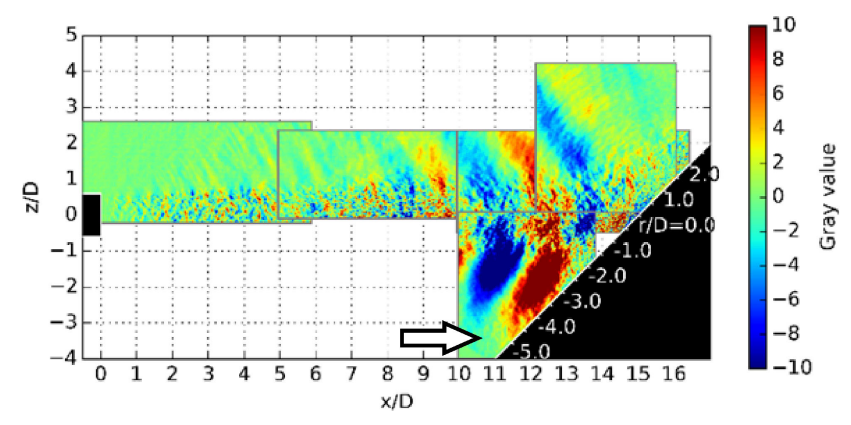

(a) $\tau=-2.40 \mathrm{~ms}$

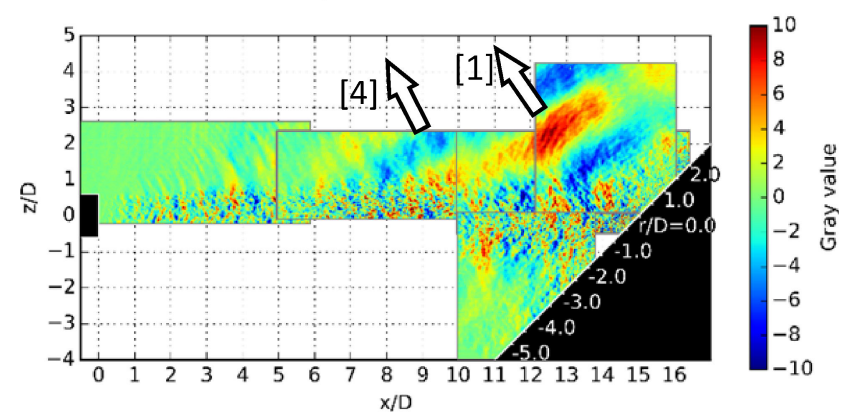

(b) $\tau=-2.00 \mathrm{~ms}$

Fig. 15. Flow phenomena images related to the acoustic waves extracted from the visualization movie using 300 trigger events of $7 \mathrm{kHz}$ (nozzleplate distance, $15 D)$.

gating in a direction approximately perpendicular to the inclined flat plate, was successfully extracted, and the following conclusions were obtained.

1. In the case of the nozzle-plate distance being $5 D$, the images extracted from the visualization movies showed that the acoustic wave was generated from the impingement region, after the arrival of density fluctuation in the free jet region at the inclined plate. Additionally, according to the results extracted from the wall surface pressure signal, this density fluctuation involved pressure fluctuation in the jet.

2. The features of this density fluctuation were similar to the Mach wave of a free jet. Therefore, it was considered that the Mach wave of the free jet region is related to the generation of acoustic waves from the impingement region. As for the mechanism of this acoustic wave generation, it cannot be explained as the reflection of the waves from the free jet region, because the source region of the acoustic wave from the impingement region was concentrated.

3. In the case of the nozzle-plate distance being $15 \mathrm{D}$, at which the OASPL took a maximum, the density fluctuation extracted was similar to that in the case of $5 D$. However, some minor differences can be found, and understanding these differences will help clarify the relation between the OASPL and nozzle-plate distance.

\section{Acknowledgments}

The authors would like to thank Ms. Chihiro Konno, an undergraduate student of the Department of Aeronautics and Astronautics, School of Engineering, The University of Tokyo, and Dr. George Kuwabara, Photron Limited, for their help during this study. 


\section{References}

1) Eldred, K. M.: Acoustic Loads Generated by the Propulsion System, NASA SP-8072, 1971.

2) Casalino, D., Barbarino, M., Genito, M., and Ferrara, V.: Hybrid Empirical/Computational Aeroacoustic Methodology for Rocket Noise Modeling, AIAA J., 47, 6 (2009), pp. 1445-1460.

3) Haynes, J. and Kenny, R.: Modifications to the NASA SP-8072 Distributed Source Method II for Ares I Lift-Off Environment Predictions, AIAA Paper 2009-3160, 2009.

4) Tsutsumi, S., Kato, S., Fukuda, K., Takaki, R., and Ui, K.: Effect of Deflector Shape on Acoustic Field of Launch Vehicle at Lift-off, AIAA Paper 2009-328, 2009.

5) Henderson, L. F.: Experiments on the Impingement of a Supersonic Jet on a Flat Plate, Zeitschrift für angewandte Mathematik und Physik ZAMP, 17, 5 (1966), pp. 553-569.

6) Donaldson, C. D. and Snedeker, R. S.: A Study of Free Jet Impingement. Part 1. Mean Properties of Free and Impinging Jets, J. Fluid Mech., 45, 2 (1971), pp. 281-319.

7) Carling, J. C. and Hunt, B. L.: The Near Wall Jet of a Normally Impinging, Uniform, Axisymmetric, Supersonic Jet, J. Fluid Mech., 66, 1 (1974), pp. 159-176.

8) Lamont, P. J. and Hunt, B. L.: The Impingement of Underexpanded Axisymmetric Jets on Perpendicular and Inclined Flat Plates, J. Fluid Mech., 100, 3 (1980), pp. 471-511.

9) Nakai, Y., Fujimatsu, N., and Fujii, K.: Experimental Study of Underexpanded Supersonic Jet Impingement on an Inclined Flat Plate, AIAA J., 44, 11 (2006), pp. 2691-2699.

10) Goto, Y., Nonomura, T., McIlroy, K., and Fujii, K.: Detailed Analysis of Flat Plate Pressure Peaks Created by Supersonic Jet Impingements, AIAA Paper 2009-1289, 2009.

11) Powell, A.: The Sound-producing Oscillations of Round Underexpanded Jets Impinging on Normal Plates, J. Acoust. Soc. Am., 83, 2 (1988), pp. 515-533.

12) Krothapalli, A., Rajkuperan, E., Alvi, F., and Lourenco, L.: Flow Field and Noise Characteristics of a Supersonic Impinging Jet, J. Fluid Mech., 392 (1999), pp. 155-181.

13) Uzun, A., Kumar, R., Hussaini, M. Y., and Alvi, F. S.: Simulation of Tonal Noise Generation by Supersonic Impinging Jets, AIAA J., 51, 7 (2013), pp. 1593-1611.

14) Henderson, B., Bridges, J., and Wernet, M.: An Experimental Study of the Oscillatory Flow Structure of Tone-producing Supersonic Impinging Jets, J. Fluid Mech., 542 (2005), pp. 115-137.

15) Dauptain, A., Gicquel, L. Y. M., and Moreau, S.: Large Eddy Simulation of Supersonic Impinging Jets, AIAA J., 50, 7 (2012), pp. $1560-1574$.

16) Risborg, A. and Soria, J.: High-speed Optical Measurements of an Underexpanded Supersonic Jet Impinging on an Inclined Plate, Proc. SPIE 7126, 71261F, 2009.

17) Nonomura, T., Goto, Y., and Fujii, K.: Aeroacoustic Waves Generated from a Supersonic Jet Impinging on an Inclined Flat Plate, Int. J. Aeroacoustics, 10, 4 (2011), pp. 401-426.

18) Tsutsumi, S., Takaki, R., Nakanishi, Y., Okamoto, K., and Teramoto, S.: Numerical Study on Acoustic Radiation from a Supersonic Jet Impinging to an Inclined Plate, AIAA Paper 2011-2922, 2011.

19) Tsutsumi, S., Takaki, R., Nakanishi, Y., Okamoto, K., and Teramoto, S.: Acoustic Generation Mechanism of a Supersonic Jet Impinging on Deflectors, AIAA Paper 2014-0882, 2014.

20) Worden, T. J., Gustavsson, J. P. R., Shih, C., and Alvi, F. S.: Acoustic Measurements of High-Temperature Supersonic Impinging Jets in Multiple Configurations, AIAA Paper 2013-2187, 2013.

21) Akamine, M., Nakanishi, Y., Okamoto, K., Teramoto, S., Okunuki, T., and Tsutsumi, S.: Acoustic Phenomena from Correctly Expanded Supersonic Jet Impinging on Inclined Plate, AIAA J., 53, 7 (2015), pp. 2061-2067.

22) Akamine, M., Nakanishi, Y., Okamoto, K., Teramoto, S., Okunuki, T., and Tsutsumi, S.: Experimental Study on Acoustic Phenomena of Supersonic Jet Impinging on Inclined Flat Plate, AIAA Paper 20140879, 2014.

23) Akamine, M., Okamoto, K., Okunuki, T., Teramoto, S., and Tsutsumi, S.: Analysis of Acoustic Signals and Flow Visualization Photographs of Supersonic Impinging Jet Using Conditional Sampling Method, 55th Conference on Aerospace Propulsion and Power, 2A09, 2015 (in Japanese).

24) Juvé, D., Sunyach, M., and Comte-Bellot, G.: Intermittency of the Noise Emission in Subsonic Cold Jets, J. Sound Vib., 71, 3 (1980), pp. 319-332.

25) Guj, G., Carley, M., and Camussi, R.: Acoustic Identification of Coherent Structures in a Turbulent Jet, J. Sound Vib., 259, 5 (2003), pp. 1037-1065.

26) Hileman, J. I., Thurow, B. S., Caraballo, E. J., and Samimy, M.: Largescale Structure Evolution and Sound Emission in High-speed Jets: Real-time Visualization with Simultaneous Acoustic Measurements, J. Fluid Mech., 544 (2005), pp. 277-307.

27) Kearney-Fischer, M., Sinha, A., and Samimy, M.: Intermittent Nature of Subsonic Jet Noise, AIAA J., 51, 5 (2013), pp. 1142-1155.

28) Torrence, C. and Compo, G. P.: A Practical Guide to Wavelet Analysis, Bull. Am. Meteorol. Soc., 79, 1 (1998), pp. 61-78.

29) Oertel, H., Seiler, F., and Srulijes, J.: Mach Wave Noise of a Supersonic Jet, 15th International Symposium of Flow Visualization, 2012.

30) Freund, J. B., Lele, S. K., and Moin, P.: Numerical Simulation of Mach 1.92 Turbulent Jet and Its Sound Field, AIAA J., 38, 11 (2000), pp. 2023-2031.

31) Papamoschou, D. and Bunyajitradulya, A.: Evolution of Large Eddies in Compressible Shear Layers, Phys. Fluids, 9, 3 (1997), pp. 756-765.

J. R. Hulka

Associate Editor 